\title{
A method to include the environmental hazard in drug prescribing
}

\author{
Staffan Castensson - Viktoria Eriksson • \\ Kristina Lindborg · Björn Wettermark
}

Received: 14 March 2008/Accepted: 11 October 2008/Published online: 27 November 2008

(C) Springer Science+Business Media B.V. 2008

\begin{abstract}
Objective Environmental classification systems for medicines have been proposed. It is important that these systems are integrated into clinical practice. Numerous studies have shown that feedback to healthcare professionals of their prescribing patterns is effective in changing behaviour. The aim of this study was to develop a method to incorporate an environmental classification for medicines in drug utilization data provided to prescribers. Drug prescribing was calculated on all pharmacy sales of medicines in ambulatory care in Stockholm, Sweden (1.9 million inhabitants) during 2006. Method Prescribing profiles were generated focusing on medicines that accounted for $90 \%$ of the volume (DU90\%) of antibiotics (ATC-group J01), drugs for musculoskeletal conditions (NSAIDs, M01A), antihypertensive agents (C03, C07, C08 and $\mathrm{C} 09$ ) and antidepressants (N06A). The medicines in these $90 \%$ segments were measured as kilograms of active
\end{abstract}

\author{
S. Castensson ( $\square)$ \\ Research and Development, National Corporation of Swedish \\ Pharmacies, Apoteket AB, 11881 Stockholm, Sweden \\ e-mail: staffan.castensson@apoteket.se \\ V. Eriksson \\ National Corporation of Swedish Pharmacies, \\ Apoteket AB, Stockholm, Sweden \\ K. Lindborg \\ Department of Clinical Immunology, \\ Karolinska University Hospital, Stockholm, Sweden \\ B. Wettermark \\ Department of Drug Management and Informatics, \\ Stockholm County Council, Stockholm, Sweden \\ B. Wettermark \\ Centre for Pharmacoepidemiology, Stockholm, Sweden
}

substance and were coded according to the classification of environmental hazard developed by Stockholm County Council and Apoteket AB. Main outcome measure Amount of pharmaceuticals dispensed $(\mathrm{kg})$ and the classification of environmental hazard for the active substances accounting for the top $90 \%$ of the total amount prescribed. Results A total of $16,800 \mathrm{~kg}$ of antibiotics, $23,400 \mathrm{~kg}$ of NSAIDs, $7,700 \mathrm{~kg}$ of various antihypertensive substances and $1,700 \mathrm{~kg}$ of antidepressants had been dispensed in the region, corresponding to $2.3,3.3,1.1$ and $0.2 \mathrm{~kg}$ of drug substance per square kilometre, respectively. A total of 12 , 3, 13 and 9 substances accounted for $90 \%$ of the total volume of antibiotics, NSAIDs, antihypertensives and antidepressants, respectively. The proportions of potentially high environmental hazard drugs were $0,7,0$ and $24 \%$, respectively. Conclusion The prescribing profiles gave an easily understandable overview for the potential environmental risk and indication for where improvement could be made. The $90 \%$ profiles were easy and inexpensive to produce using available sales data and may be a valuable tool to increase the awareness of the environmental aspects among prescribing doctors.

Keywords Environmental hazard · Drug prescribing · Sweden · Drug utilization

\section{Impact of findings on practice}

- The method can support policy decisions in guidelines and formularies to recommend less environmentally harmful pharmaceuticals.

- The method may be useful to improve prescribers' environmental awareness in their choice of drugs. 


\section{Introduction}

Access to clean water is a prerequisite for good health. Most drugs consumed are subsequently secreted in the urine, in an unchanged condition or as metabolites. In urban areas the compounds will reach sewage treatment plants and sometimes even rivers, lakes and ground water. Many drug molecules entering sewage treatment plants will travel through without being degraded or removed by the cleaning processes. Since the utilization of chemicals, including pharmaceuticals, is continuously growing, there is an increasing risk from chemical substances to contaminate our food and water supply within the environmental cycles. Environmental persistence in combination with bioaccumulation means that pharmaceutical residues are an increasing problem in diverse environmental compartments and lead to increased concerns about the ecotoxicity of pharmaceutical products $[1,2]$.

A number of studies have been published that show traces of medicines in water from sewage treatment plants as well as in lakes and rivers. Medicines that were reported to have a high concentration in the environment included various antihypertensives and non-steroidal anti-inflammatory drugs (NSAIDs) [3-6]. Some antibiotics and hormonal preparations have also been detected [7, 8].

Health protection and environmental protection are societal aims which usually accompany each other. However, the use of pharmaceuticals shows that both aims can become contradictory. The Swedish parliament has determined certain environmental objectives, including; that by 2010 there should be information on the environmental characteristics for all manufactured or extracted chemical substances on the market [9]. In pursuance of these environmental objectives the Swedish Medical Products Agency, has identified a number of important actions to be taken to protect the environment (Table 1) [10].

Stockholm County Council (which has responsibility for the provision of healthcare in the Swedish metropolitan region of Stockholm) has the political vision to eliminate the emission of harmful medical substances to the ground,

Table 1 Measures regarding environmental aspects of pharmaceuticals proposed by the Swedish Medical Products Agency [10]

Increased basic knowledge

Increased consideration of environmental aspects in the EU legislation on pharmaceutical products

More and improved environmental risk assessments

Compilation of and electronic access to data

Continuous monitoring of the flows of pharmaceuticals

Education and information to stimulate environmental awareness

Reduce disposal and improve waste-handling of pharmaceutical products sea and air. For most environmentally hazardous medicinal products in effluent from sewage treatment plants or in surface water, the current aim is to achieve lower levels in 2011 as compared to 2005 measurements. Consequently, Stockholm County Council will favour pharmaceuticals that are not harmful to the environment and work to influence the pharmaceutical industry to take environmental issues into greater account. This is reflected in the treatment guidelines issued by the regional Drug and Therapeutics Committee where drugs are recommended in preference where the environmental hazard is lower after the standard tests of safety, efficacy and cost effectiveness have been taken into account [11].

A classification of environmental hazard for pharmaceuticals has been developed by Stockholm County Council and Apoteket AB (The National Corporation of Swedish Pharmacies) to express the inherent environmentally damaging characteristics of the active pharmaceutical ingredient $[12,13]$. The classification is based on the assessment of:

- Persistence $(\mathrm{P})$ - ability to resist degradation in the aquatic environment

- Bioaccumulation (B)—accumulation in adipose tissue of aquatic organisms

- Toxicity (T) — the potential to poison aquatic organisms

Each of these characteristics is assigned a numerical value (0-3). The sum of these values constitutes the PBT index for the substance. The PBT index can assume values between 0 and 9 .

Since 2005, information about the environmental classification for the most commonly prescribed medicines in ambulatory care is printed in a booklet distributed to all primary healthcare centres and hospital clinics in the county. The booklet is updated annually and the information is also presented on the county's website for drug information [14]. However, it is known that simple diffusion or dissemination of printed material is seldom effective in changing prescribing doctors' behaviour [15, 16]. To promote effective change it is important to combine evidence based information with other methods such as interactive education or feedback on prescribing patterns. A method that has been shown to be useful in implementing guidelines is the Drug Utilization $90 \%$ (DU90\%) method providing feedback on the medicines accounting for $90 \%$ of the utilization and adherence to formulary recommendations within the section. The $90 \%$ level is focusing on the quality of the bulk of the prescribing whilst allowing some leeway for individual variation $[17,18]$. When adding an environmental hazard dimension, these profiles could provide a useful tool to increase prescribing doctors' environmental awareness of their prescribing practice. 


\section{Aim of the study}

The aim of this study was to develop a method to include environmental classification for medicines in drug utilization data.

\section{Method}

Data on sales of medicines in ambulatory care dispensed in 2006 were obtained from Apoteket AB (National Corporation of Swedish Pharmacies). The data contained both nonprescription (OTC) sales and all prescriptions dispensed to the Swedish population, regardless of reimbursement status. We selected antibiotics (ATC-group J01), drugs for musculoskeletal conditions (NSAIDs including glucosamine) (M01A), antihypertensive agents (diuretics, beta blockers, calcium channel blockers and drugs acting on the reninangiotensin system) (C03, C07, C08, C09) and antidepressants (N06A) dispensed in the region of Stockholm, Sweden.

The data were analysed by active substance, defined as all products marketed under a single ATC group [19] and measured in number of kilograms of the substance. Products containing fixed combinations were divided and calculated separately for each substance. The total utilization for the period for each ATC-group was determined. We then calculated the number of drugs that accounted for $90 \%$ of the total weight in the group (the area under the curve), i.e. DU90\%.

The total amount dispensed was divided by the surface area in the region excluding the Baltic Sea, i.e. $7,169 \mathrm{~km}^{2}$, and the population (1.9 million inhabitants) [20]. These denominators were chosen to asses the intensity of environmental emission, both from a geographical and a population perspective. The drug utilization profiles also contained the number of Defined Daily Doses (DDDs) and the number of DDDs per thousand inhabitants per day (DDD/TID) as a therapeutic measure of exposure [19].

The drugs prescribed were coded into three groups according to the classification of environmental hazard developed by Stockholm County Council and Apoteket AB [12-14]. The classification is based on an evaluation of each substance's persistence, potential bioaccumulation and toxicity. Indexing is provided on a scale of 0-9 and can be seen as an indication of the active substance's inherent danger to the environment based on a sum of numeric values for the active substance (Table 2).

An active substance that is readily biodegradable, has low toxicity and does not bioaccumulate receives an environmental index value of zero. A substance that is not readily biodegradable, has very high toxicity and is potentially bio accumulating receives an environmental index value of 9. Substances with incomplete data have
Table 2 Principles for the assignment of numerical values in the environmental hazard classification developed by Stockholm County Council and Apoteket AB [12, 13]

\begin{tabular}{ll}
\hline Persistence (readily biodegradable) & 0 \\
Yes & 3 \\
No & 3 \\
Bioaccumulation (potentially bioaccumulating) & 0 \\
Yes & \\
No & 3 \\
Toxicity & 2 \\
Very high toxicity & 1 \\
High toxicity & 0 \\
Moderate toxicity & \\
Low toxicity & \\
\hline
\end{tabular}

Note: The PBT denotations used by Stockholm County Council are not to be mixed up with the well established denotations PBT and vPvB used for identifying substances according to TGD 2003 [21]. The PBT and $\mathrm{vPvB}$ criteria and their application are described by the Danish EPA [22]

also been assigned higher values; consequently the indexing could also mean a compensation for the lack of data applying a precautionary principle.

The classification of environmental hazard according to Stockholm County Council has not been scientifically validated. It can be regarded as a model elucidating that drugs can be grouped in a systematic way with reference to hazard based on available scientific data. However, no absolute stratification is accomplished by the index value as these are not mutually comparable. This implies that comparing two or more drugs with reference to environmental hazard will require going back to the scientific data describing the hazard of a specific substance.

In this study, we have simplified the classification for each substance one step further and marked the class in the drug utilization profiles accordingly. Substances with a PBT index of 7-9 were made black, 4-6 dark grey and 0-3 light grey, respectively, to illustrate potential high, medium and low hazard. Drugs without any classification were presented as striped. The proportions of high/medium/low risk drugs were assessed by calculating the amount of kilograms of the substances in each group divided by the total weight of the $90 \%$-segment.

\section{Results}

A total of $16,800 \mathrm{~kg}$ of antibiotics, $23,400 \mathrm{~kg}$ of NSAIDs, $7,700 \mathrm{~kg}$ of various antihypertensive substances and $1,700 \mathrm{~kg}$ of antidepressants had been dispensed in the region during 2006. This corresponds to 8.8, 12.3, 4.0 and $0.9 \mathrm{~kg} / 1000$ inhabitants, respectively, or $2.3,3.3,1.1$ and $0.2 \mathrm{~kg} / \mathrm{km}^{2}$. 
In the antibiotics group there were a total of 58 substances. Twelve of these accounted for $90 \%$ of the utilization. Out of these 12 , there were six substances, that included the three most used (phenoxymethylpenicillin, methenamine and flucloxacillin) that were not classified (Fig. 1). All the antibiotics that have been classified were considered having a potential medium environmental hazard (PBT 4-6). Phenoxymethylpenicillin was the most used of the antibiotics. It accounted for $6,300 \mathrm{~kg}, 37.8 \%$ of the total and 4.5 DDD/TID.

In total, 19 different drugs for musculoskeletal conditions were used. Three of them (ibuprofen, glucosamine and naproxen) accounted for $90 \%$ of the utilization (Fig. 2). Ibuprofen and glucosamine were both available as OTC during the period. The proportion of non-prescription sales was $83 \%$ and 19\%, respectively. Since both ibuprofen and glucosamine were considered with low environmental hazard, the profile was rather good from an environmental perspective (Fig. 2). However, naproxen, the third most used substance, is considered to be potentially harmful for the environment (PBT 7-9).

The antihypertensive groups contained a total of 44 different substances, out of which 13 accounted for $90 \%$ of the amount. Two antihypertensive substances, verapamil and spironolactone, had not yet been classified (Fig. 3). The most used substance was metoprolol (2,300 kg). Four substances in the DU90\% segment (furosemide, losartan, propranolol and enalapril) were suggested to be associated with potential low environmental hazard (PBT 0-3) (Fig. 3). The remaining classified substances had a potential medium environmental hazard (PBT 4-6).

A total of 22 different antidepressants were used in the study period. All nine antidepressants within the DU90\% segment had an environmental classification (Fig. 4). No single substance used was considered to have a potential low environmental risk. Two substances (citalopram and mirtazapine), together accounted for one quarter of the total volume within the DU90\% segment and were considered to be associated with a potential high environmental hazard.

\section{Discussion}

The results show the feasibility of combining sales statistics of drugs with classification of environmental hazard. The proposed method may be a useful tool to bring environmental aspects into the prescribing decisions of healthcare professionals. The DU90\% profiles were inexpensive to produce and were based on readily available data for dispensed medicines. The sales data were complete for the therapeutic areas selected including all drugs dispensed in ambulatory care regardless of reimbursement and prescription status. Further refinement may be done depending on the data available. It is possible to produce
Fig. 1 DU90\% profile for antibiotics (J01) for Stockholm County, Sweden 2006

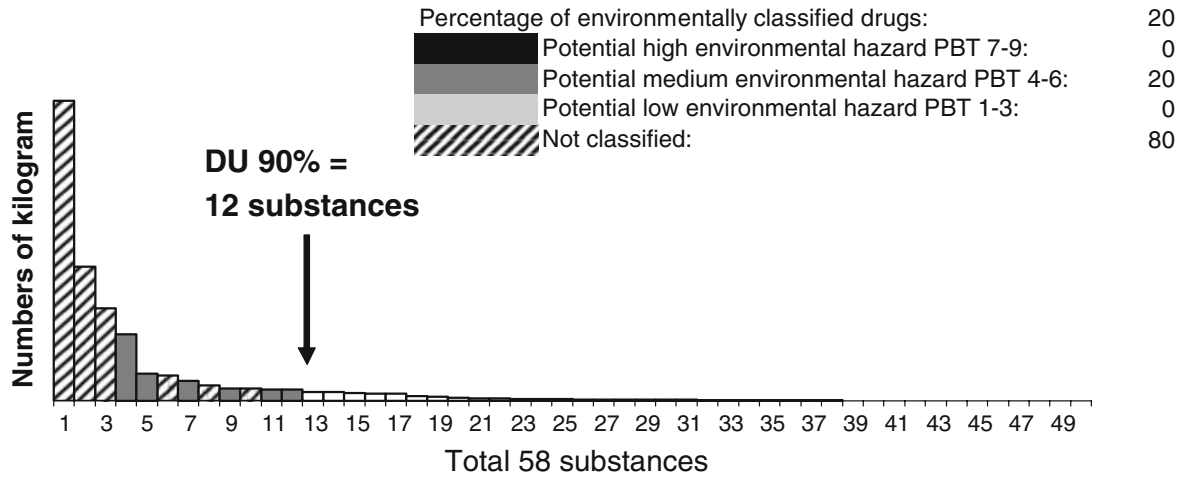

\begin{tabular}{|c|c|c|c|c|c|c|c|c|}
\hline & Substance & (DDD) & $\mathbf{K g}$ & $\%$ TOT & $\mathrm{Kg} / \mathrm{km} 2$ & $\begin{array}{c}\mathrm{Kg} / 1000 \\
\text { inhabitant }\end{array}$ & DDD & DDD/TID \\
\hline 1 & Phenoxymethylpenicillin & $2 \mathrm{~g}$ & 6348 & $37,8 \%$ & 0,89 & 3,3 & 3174007 & 4,5 \\
\hline 2 & Methenamine & $2 \mathrm{~g}$ & 2833 & $16,9 \%$ & 0,40 & 1,5 & 1416679 & 2,0 \\
\hline 3 & Flucloxacillin & $2 \mathrm{~g}$ & 1946 & $11,6 \%$ & 0,27 & 1,0 & 972770 & 1,4 \\
\hline 4 & Amoxicillin & $1 \mathrm{~g}$ & 1400 & $8,3 \%$ & 0,20 & 0,7 & 1400320 & 2,0 \\
\hline 5 & Ciprofloxacin & $0.5 \mathrm{~g} / 1 \mathrm{~g}$ & 567 & $3,4 \%$ & 0,08 & 0,3 & 571616 & 0,8 \\
\hline 6 & Lymecycline & $0.6 \mathrm{~g}$ & 538 & $3,2 \%$ & 0,07 & 0,3 & 895900 & 1,3 \\
\hline 7 & Erythromycin & $1 g / 2 g$ & 425 & $2,5 \%$ & 0,06 & 0,2 & 379042 & 0,5 \\
\hline 8 & Cefadroxil & $2 \mathrm{~g}$ & 319 & $1,9 \%$ & 0,04 & 0,2 & 159445 & 0,2 \\
\hline 9 & Sulfamethoxazole & $2 \mathrm{~g}$ & 256 & $1,5 \%$ & 0,04 & 0,1 & 160214 & 0,2 \\
\hline 10 & Clindamycin & $1.2 \mathrm{~g} / 1.8 \mathrm{~g}$ & 254 & $1,5 \%$ & 0,04 & 0,1 & 206689 & 0,3 \\
\hline 11 & Tetracycline & $1 \mathrm{~g}$ & 233 & $1,4 \%$ & 0,03 & 0,1 & 232671 & 0,3 \\
\hline 12 & Trimethoprim & $0.4 \mathrm{~g}$ & 231 & $1,4 \%$ & 0,03 & 0,1 & 533939 & 0,8 \\
\hline \multirow{2}{*}{\multicolumn{2}{|c|}{$\begin{array}{cc}\text { DU } 90 \% & 1-12 \\
13-58 & \end{array}$}} & & 15350 & 91,3\% & 2,14 & 8,0 & & \\
\hline & & & 1464 & $8,7 \%$ & 0,20 & 0,8 & & \\
\hline \multicolumn{3}{|c|}{ TOTAL $\quad 1-58$} & 16813 & $100,0 \%$ & 2,35 & 8,8 & & \\
\hline
\end{tabular}


Fig. 2 DU90\% profile for NSAIDs (including glucosamine) (M01A) for Stockholm County, Sweden 2006

Fig. 3 DU90\% profile for antihypertensives (C03, C07, C08 and C09) for Stockholm County, Sweden 2006

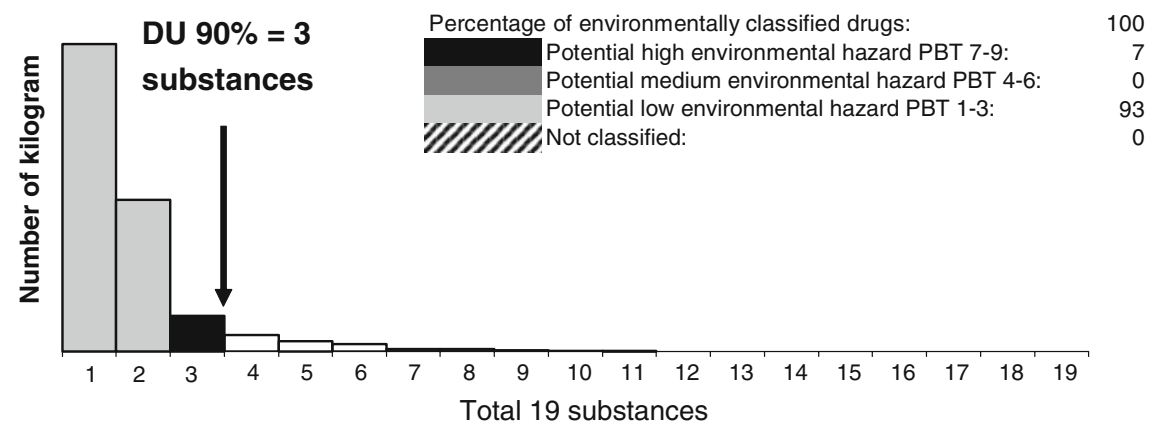

\begin{tabular}{|c|c|c|c|c|c|c|c|c|}
\hline & Substance & (DDD) & $\mathbf{K g}$ & \% TOT & $\mathrm{Kg} / \mathrm{km} 2$ & $\begin{array}{c}\mathrm{Kg} / 1000 \\
\text { inhabitant }\end{array}$ & DDD & DDD/TID \\
\hline & Ibuprofen & $1.2 \mathrm{~g}$ & 13460 & $57,4 \%$ & 1,88 & 7,0 & 11327831 & 16,2 \\
\hline & Glucosamine & $1.5 \mathrm{~g}$ & 6639 & $28,3 \%$ & 0,93 & 3,5 & 4426193 & 6,3 \\
\hline & Naproxen & $0.5 \mathrm{~g}$ & 1554 & $6,6 \%$ & 0,22 & 0,8 & 3107301 & 4,5 \\
\hline DU $90^{\circ}$ & $1-3$ & & 21653 & $92,4 \%$ & 3,02 & 111,3 & & \\
\hline $4-1$ & & & 1793 & $7,6 \%$ & 0,25 & 0,9 & & \\
\hline TOTA $\bar{L}$ & L $\quad 1-19$ & & 23446 & $100,0 \%$ & 3,27 & 12,3 & & \\
\hline
\end{tabular}

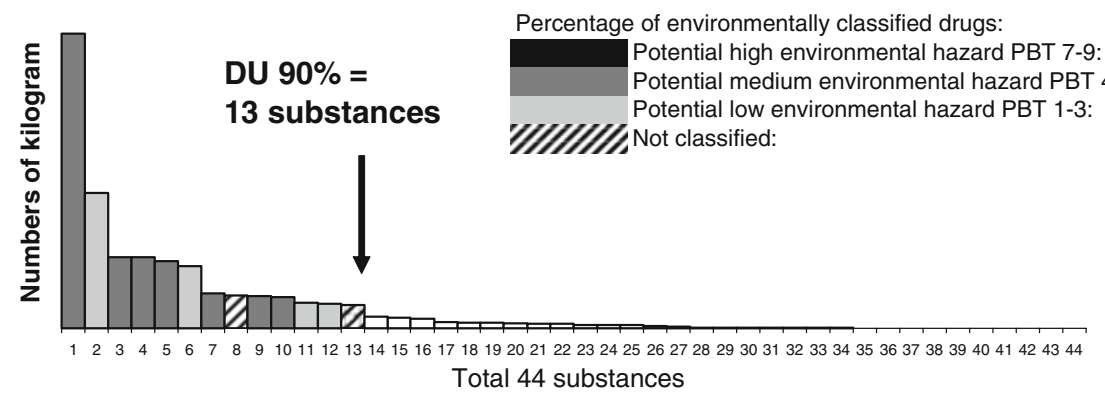
Total 44 substances

\begin{tabular}{|c|c|c|c|c|c|c|c|c|}
\hline & Substance & (DDD) & $\mathbf{K g}$ & $\%$ TOT & $\mathrm{Kg} / \mathbf{k m} 2$ & $\begin{array}{c}\mathrm{Kg} / 1000 \\
\text { inhabitant }\end{array}$ & DDD & DDD/TID \\
\hline 1 & Metoprolol & $0.15 \mathrm{~g}$ & 2308 & $30,0 \%$ & 0,32 & 1,2 & 15891268 & 22,8 \\
\hline 2 & Furosemide & $40 \mathrm{mg}$ & 1059 & $13,8 \%$ & 0,15 & 0,6 & 26474862 & 37,9 \\
\hline 3 & Atenolol & $75 \mathrm{mg}$ & 557 & $7,2 \%$ & 0,08 & 0,3 & 7430076 & 10,6 \\
\hline 4 & Hydrochlorothiazide & $25 \mathrm{mg}$ & 556 & $7,2 \%$ & 0,08 & 0,3 & 17929096 & 25,7 \\
\hline 5 & Irbesartan & $0.15 \mathrm{~g}$ & 524 & $6,8 \%$ & 0,07 & 0,3 & 2930088 & 4,2 \\
\hline 6 & Losartan & $50 \mathrm{mg}$ & 487 & $6,3 \%$ & 0,07 & 0,3 & 8974519 & 12,9 \\
\hline 7 & Valsartan & $80 \mathrm{mg}$ & 273 & $3,5 \%$ & 0,04 & 0,1 & 2910515 & 4,2 \\
\hline 8 & Verapamil & $0.24 \mathrm{~g}$ & 255 & $3,3 \%$ & 0,04 & 0,1 & 1072476 & 1,5 \\
\hline 9 & Sotalol & $0.16 \mathrm{~g}$ & 250 & $3,3 \%$ & 0,03 & 0,1 & 1565615 & 2,2 \\
\hline 10 & Diltiazem & $0.24 \mathrm{~g}$ & 243 & $3,2 \%$ & 0,03 & 0,1 & 1014316 & 1,5 \\
\hline 11 & Enalapril & $10 \mathrm{mg}$ & 196 & $2,6 \%$ & 0,03 & 0,1 & 18171392 & 26,0 \\
\hline 12 & Propranolol & $0.16 \mathrm{~g}$ & 191 & $2,5 \%$ & 0,03 & 0,1 & 1192694 & 1,7 \\
\hline 13 & Spironolactone & $75 \mathrm{mg}$ & 182 & $2,4 \%$ & 0,03 & 0,1 & 2422197 & 3,5 \\
\hline \multicolumn{2}{|c|}{ DU $90 \% \quad 1-13$} & & 7082 & $92,1 \%$ & 0,99 & 3,7 & & \\
\hline \multicolumn{2}{|c|}{$14-44$} & & 605 & $7,9 \%$ & 0,08 & 0,3 & & \\
\hline TOTA & $\begin{array}{l}\mathrm{L} \quad 1-44 \\
\end{array}$ & & 7687 & $100,0 \%$ & 1,07 & 4,0 & & \\
\hline
\end{tabular}

similar prescribing profiles for primary care practices, hospital clinics or populations based on various population levels from municipalities up to a region or even a country. The selection may be made for a certain pharmacological group (ATC), but environmental risk profiles may also be produced for the whole range of pharmaceutical products prescribed to provide an overall environmental risk assessment. These profiles represent one way to find the most important areas of improvement. However, profiles restricted to a certain pharmacological group are more intuitive from a therapeutic point of view since in this scenario the drugs may be interchangeable.

Rational drug therapy can be defined as "Patients receive medication appropriate to their medical needs, in doses meeting their own individual requirements, for an adequate period of time and at the lowest cost to them and the community" [23]. We propose that environmental concern should be included in this definition. However, such environmental concern should always be considered in combination with other important aspects as efficacy, safety 
Fig. 4 DU90\% profile for antidepressants (N06A) for Stockholm County, Sweden 2006

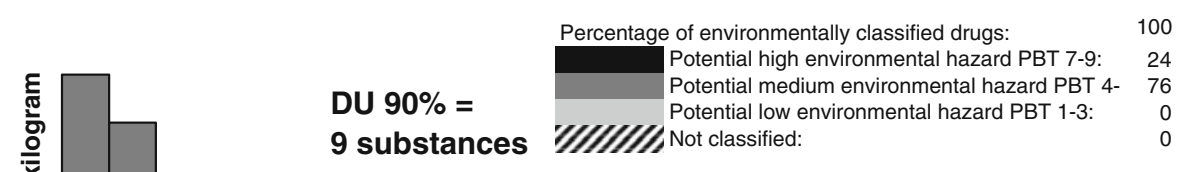

24
76
0
0

\begin{tabular}{|c|c|c|c|c|c|c|c|c|}
\hline & Substance & (DDD) & Kg & $\%$ TOT & $\mathrm{Kg} / \mathrm{km} 2$ & $\begin{array}{c}\mathrm{Kg} / 1000 \\
\text { inhabitant }\end{array}$ & DDD & DDD/TID \\
\hline 1 & Sertraline & $50 \mathrm{mg}$ & 455 & $27,4 \%$ & 0,06 & 0,24 & 9105589 & 13,0 \\
\hline 2 & Venlafaxine & $0.1 \mathrm{~g}$ & 381 & $22,9 \%$ & 0,05 & 0,20 & 3812492 & 5,5 \\
\hline 3 & Citalopram & $20 \mathrm{mg}$ & 269 & $16,2 \%$ & 0,04 & 0,14 & 13474641 & 19,3 \\
\hline 4 & Mirtazapine & $30 \mathrm{mg}$ & 105 & $6,3 \%$ & 0,01 & 0,05 & 3502759 & 5,0 \\
\hline 5 & Amitriptyline & $75 \mathrm{mg}$ & 95 & $5,7 \%$ & 0,01 & 0,05 & 1262560 & 1,8 \\
\hline 6 & Fluoxetine & $20 \mathrm{mg}$ & 65 & $3,9 \%$ & 0,01 & 0,03 & 3232951 & 4,6 \\
\hline 7 & Clomipramine & $0.1 \mathrm{~g}$ & 62 & $3,7 \%$ & 0,01 & 0,03 & 621221 & 0,9 \\
\hline 8 & Duloxetine & $60 \mathrm{mg}$ & 62 & $3,7 \%$ & 0,01 & 0,03 & 1031893 & 1,5 \\
\hline 9 & Moclobemide & $0.3 \mathrm{~g}$ & 42 & $2,5 \%$ & 0,01 & 0,02 & 138892 & 0,2 \\
\hline DU 90 & 10 & & 151536 & 92,4\% & "0,21 & 0,80 & & \\
\hline $10-$ & -22 & & 127 & $7,6 \%$ & 0,02 & 0,07 & & \\
\hline TOTA & L $\quad 1-22$ & & 1663 & $100,0 \%$ & 0,23 & 0,87 & & \\
\hline
\end{tabular}

and cost-effectiveness to optimize drug therapy. We believe that drug utilization profiles focusing on environmental hazard have great value in pharmaceutical policymaking, e.g. as a basis for drug selection to formularies and guidelines, such as the regional Drug and Therapeutics Committee guidelines used in Stockholm [11].

It is important to improve the utilization of antibiotics. It has been shown that the total utilization of antibiotics is correlated to antimicrobial resistance [24]. The WHO has pointed out antimicrobial resistance as a main concern for global health [25]. Studies have shown a considerable variation between countries of the utilization of antibiotics with Sweden reporting a relatively low utilization [26]. In our study, the choice of antibiotic substances seemed to be rational from a therapeutic point of view since the most used antibiotic was the narrow spectrum phenoxymethylpenicillin. The environmental aspects are uncertain since the most used antibiotics were not classified. Further efforts need to be taken to assess the environmental hazard for those substances and drugs of other therapeutic areas where no data is currently available.

The utilization of NSAIDs seemed to be appropriate from an environmental point of view. Ibuprofen, the most used NSAID, should be the preferred choice among NSAIDs also due to its relatively low gastrointestinal toxicity [27]. However, questions may be raised about the total level of use of NSAIDs and the high use of glucosamine. The latter has a poor therapeutic value and should consequently not be used, although it seems to be relatively harmless for the environment [28].
There was no obvious potential for environmental improvement in the utilization of antihypertensives. The different medicines represent different drug classes that are all recommended for certain patient groups. Furthermore, the drugs are not only used for the treatment of hypertension but for a variety of other medical conditions, e.g. heart failure, angina pectoris and secondary prevention after myocardial infarction. Although the profile indicates that furosemide, losartan, propranolol and enalapril may be preferable from an environmental point of view, these drugs may not be recommended as alternatives due to differences in licensed indication, safety profile and costeffectiveness compared to the alternatives. However, questions may be raised about the high utilization of beta blockers, since their effectiveness has been under discussion in recent years [29]. A higher use of ACE-inhibitors and thiazides (where therapeutically indicated) may therefore be recommended since they have a broad range of licensed indications, a comparatively low price and a good environmental profile. Also, it is important to emphasize that many reports have shown that there is an under treatment of hypertension [30].

Since the drug potency varies, some drugs with a high utilization in the population, e.g. the calcium channel blockers felodipine and amlodipine, generated low amounts when measured in kilograms and thus did not appear within the DU90\% segment for the antihypertensives. This may to some extent reduce the external validity of the method for prescribers. A potential solution could be to extend the cut off point to, e.g., 95\%. It can also be handled by 
complementing the DU90\% environmental profiles with traditional profiles using DDD as the measure of exposure.

All antidepressants were environmentally classified, two substances being associated with a potentially high environmental risk. There is a limited potential for improvement in the utilization of antidepressant drugs, since there are no antidepressants associated with a potential low environmental risk. However, citalopram may be substituted with other serotonin selective reuptake inhibitors since they have shown to be equally effective [31].

As discussed above, the environmental prescribing profiles highlighted several areas for improvement. However, the prescribing profiles should be applied as a recommendation for improvement on a population level. It is important to emphasize that rational drug therapy implies a rational decision for each individual patient taking into account the efficacy, tolerability, and potential for interactions of each specific drug. Consequently, there may be reasons to deviate from general guidelines for certain patients. The credibility of the proposed method is dependent on the validity of the DU90\% method as well as validity of the classification of environmental hazard. The DU90\% method has been validated in a large number of studies and is increasingly used in many countries to assess the quality of prescribing [32]. The low validity of the classification model adopted in this study will hamper a more general application of the proposed method. Nevertheless, being knowledgeable about its drawbacks it can still serve as a driver for a better environmental outcome of drug use.

The legislation for new drugs to be approved for use in humans has been continuously strengthened during the last decades. The thalidomide tragedy tightened the requirements to provide safety data and in recent years, most countries require manufacturers to provide evidence that new drugs are more cost-effective than existing agents to achieve reimbursement. Environmental concern does not yet influence any approval or reimbursement decision for human medicines [33]. This may change in the future. Regardless of how this development will proceed, robust and convenient methods will still be needed to guide healthcare providers to use those medicines available on the market more rationally. This does not imply a 'one size fit all' thinking but more an 'added value' to the individual drug choice by a systematic inclusion of scientifically based evidence that can be generally applied. The method we propose supports these aims of bringing the environmental dimension into the drug selection process.

\section{Conclusion}

The DU90\% environmental profiles gave an easily understandable overview of the potential prevailing environmental risk and potentials for improvement in drug prescribing. The profiles were easy and inexpensive to produce using available sales data, and may be a valuable tool to increase the awareness of an environmental impact of the prescribing decision.

Acknowledgements We gratefully acknowledge Hans J:son Moosberg at the Division of Statistics at the National Corporation of Swedish Pharmacies for valuable assistance in the data analysis and Colin Richman, UK, for valuable comments.

Funding The study was supported by the National Corporation of Swedish Pharmacies, Apoteket AB.

Conflicts of interest No conflicts of interest.

\section{References}

1. Kümmerer K, editor. Pharmaceuticals in the environment. Sources, fate, effects and risks. 2nd ed. Berlin, Heidelberg, New York: Springer; 2004. ISBN 3-540-21342-2.

2. Apoteket AB (The National Corporation of Swedish Pharmacies), Stockholm County Council, Stockholm University. Environment and pharmaceuticals. Stockholm, Sweden: Apoteket AB; 2006. ISBN 91-85574-55-4. Cited 2008 Jan 18. Available from: http://www2.apoteket.se/NR/rdonlyres/FAB1EBDB-319A-4B4595DA-CB4D863FE525/0/EnvironmentandPharmaceuticals.pdf.

3. Ashton $\mathrm{D}$, Hilton $\mathrm{M}$, Thomas KV. Investigating the environmental transport of human pharmaceuticals to streams in the United Kingdom. Sci Total Environ. 2004;333:167-84. doi:10.1016/ j.scitotenv.2004.04.062.

4. Halling-Sørensen B, Nors Nielsen S, Lanzky PF, Ingerslev F, Holten Lützhøft HC, Jørgensen SE. Occurrence, fate and effects of pharmaceutical substances in the environment-a review. Chemosphere. 1998;36:357-93. doi:10.1016/S0045-6535(97)00354-8.

5. Nikolaou A, Meric S, Fatta D. Occurrence patterns of pharmaceuticals in water and wastewater environments. Anal Bioanal Chem. 2007;387:1225-34. doi:10.1007/s00216-006-1035-8.

6. Zuccato E, Castiglioni S, Fanelli R, Reitano G, Bagnati R, Chiabrando $\mathrm{C}$, et al. Pharmaceuticals in the environment in Italy: causes, occurrence, effects and control. Environ Sci Pollut Res Int. 2006;13:15-21. doi:10.1065/espr2006.01.004.

7. Kolpin DW, Furlong ET, Meyer MT, Thurman EM, Zaugg SD, Barber LB, et al. Pharmaceuticals, hormones, and other organic wastewater contaminants in U.S. streams, 1999-2000: a national reconnaissance. Environ Sci Technol. 2002;36:1202-11. doi: 10.1021/es011055j.

8. Kümmerer K. Significance of antibiotics in the environment. J Antimicrob Chemother. 2003;52:5-7. doi:10.1093/jac/dkg293.

9. Environmental Objectives Secretariat. Environmental objectives portal. Cited 2008 Jan 21. Available from: http://www.miljomal. nu/english/english.php.

10. Miljöpåverkan från läkemedel samt kosmetiska och hygieniska produkter. Rapport från Läkemedelsverket. Augusti 2004. (Environmental impact of drugs and of cosmetic and hygienic products. Report from Swedish Medical Products Agency. August 2004.) Cited 2008 Jan 21. Available from: http://www. lakemedelsverket.se/upload/Om\%20LV/publikationer/040824_ miljouppdraget-rapport.pdf.

11. Wettermark B, Godman B, Andersson K, Gustafsson LL, Haycox A, Bertele V. Recent national and regional reforms in Sweden- 
implications for pharmaceutical companies in Europe. Pharmacoeconomics. 2008;26:537-50.

12. Wennmalm $\AA$, Gunnarsson B. Public health care management of water pollution with pharmaceuticals: environmental classification and analysis of pharmaceutical residues in sewage water. Drug Inf J. 2005;39:291-7.

13. Stockholm County Council (homepage on the Internet). About classification. Updated: 2007-07-05; cited 2008 Jan 21. Available from: http://www.janusinfo.se/imcms/servlet/GetDoc?meta_id=7239.

14. Stockholm County Council (homepage on the Internet). Environmentally classified pharmaceuticals. Updated: 2007-12-11; cited 2008 Jan 21. Available from: http://www.janusinfo.se/ imcms/servlet/GetDoc?meta_id=7238.

15. Bero LA, Grilli R, Grimshaw JM, Harvey E, Oxman AD, Thomson MA. Closing the gap between research and practice: an overview of systematic reviews of interventions to promote the implementation of research findings. The Cochrane Effective Practice and Organization of Care Review Group. BMJ. 1998; 317:465-8.

16. Grimshaw JM, Thomas RE, MacLennan G, Fraser C, Ramsay $\mathrm{CR}$, Vale L, et al. Effectiveness and efficiency of guideline dissemination and implementation strategies. Health Technol Assess. 2004;8(6):iii-iv, 1-72.

17. Wettermark B, Pehrsson A, Jinnerot D, Bergman U. Drug utilisation $90 \%$ profiles-a useful tool for quality assessment of prescribing in primary health care in Stockholm. Pharmacoepidemiol Drug Saf. 2003;12:499-510. doi:10.1002/pds.852.

18. Wettermark B, Haglund K, Gustafsson LL, Persson PM, Bergman $\mathrm{U}$. A study of adherence to drug recommendations by providing feedback of outpatient prescribing patterns to hospital specialists. Pharmacoepidemiol Drug Saf. 2005;14:579-88. doi:10.1002/ pds.1098.

19. Collaboration WHO. Center for drug statistics methodology (homepage on the Internet). Guidelines for ATC classification and DDD assignment. Oslo; 2007. Cited 2008 Jan 21. Available from: www.whocc.no.

20. Statistics Sweden. Cited 2008 June 27. Available from: http://www.h.scb.se/kommunfakta/pyramider/pyra_frame.asp? region $=01$.

21. European Chemicals Bureau (ECB). Technical Guidance Document on Risk Assessment in support of Commission Directive 93/ 67/EEC on Risk Assessment for new notified substances and Commission Regulation (EC) No 1488/94 on Risk Assessment for existing substances and Directive 98/8/EC of the European Parliament and of the Council concerning the placing of biocidal products on the market. Part I. EUR 20418 EN/1. Luxembourg;
2003. Cited 2008 Sept 24. Available from: http://ecb.jrc.it/ Documents/TECHNICAL_GUIDANCE_DOCUMENT/EDITION_ 2/tgdpart1_2ed.pdf.

22. Tyle H, Larsen HS, Wedebye EB, Sijm D, Pedersen Krog T, Niemelä J. Identification of potential PBTs and vPvBs by use of QSARs. Copenhagen; 2002. Cited 2008 Sept 24. Available from: http://ecb.jrc.it/classlab/otherdocs/QSAR_PBT_final_clean.doc.

23. World Health Organization (WHO). The rational use of drugs. WHO Report of the Conference of Experts, Nairobi. Geneva, Switzerland: World Health Organization; 1985.

24. Bronzwaer SL, Cars O, Buchholz U, Mölstad S, Goettsch W, Veldhuijzen IK, et al. European antimicrobial resistance surveillance system. A European study on the relationship between antimicrobial use and antimicrobial resistance. Emerg Infect Dis. 2002;8:278-82.

25. Kaplan W, Laing R. Priority medicines for Europe and the world project. A public health approach to innovation. Geneva: WHO; 2004.

26. Goossens H, Ferech M, Vander Stichele R, Elseviers M. Outpatient antibiotic use in Europe and association with resistance: a cross-national database study. Lancet. 2005;365:579-87.

27. Henry D, Lim LLY, Garcia Rodrigues LA, Perez Gutthann S, Carson JL, Griffin M, et al. Variability in risk of gastrointestinal complications with individual non-steroidal anti-inflammatory drugs: results of a collaborative meta-analyses. BMJ. 1996;312: 1563-6.

28. Towheed TE, Maxwell L, Anastassiades TP, Shea B, Houpt J, Robinson V, et al. Glucosamine therapy for treating osteoarthritis. Cochrane Database Syst Rev. 2005;2:CD002946.

29. Lindholm LH, Carlberg B, Samuelsson O. Should beta blockers remain first choice in the treatment of primary hypertension? A meta-analysis. Lancet. 2005;366:1545-53. doi:10.1016/S01406736(05)67573-3.

30. Swedish Council on Technology Assessment in Health Care (SBU). Moderately elevated blood pressure. SBU report 170, 2004. Stockholm; 2004.

31. Swedish Council on Technology Assessment in Health Care (SBU). Treatment of depression. A systematic review. Report number 166 (2 vol). 2004. ISBN 91-87890-88-7.

32. Wettermark B. Drug utilization $90 \%$ - using aggregate drug statistics for the quality assessment of prescribing. 2004. ISBN 91-7140-048-6.

33. EMEA. Guideline on the environmental risk assessment of medicinal products for human use EMEA/CHMP/SWP/4447/00. London; 2006. Available from: http://www.emea.europa.eu/pdfs/ human/swp/444700en.pdf. 